\title{
Specify Other Performance Status Scale: Timing
}

National Cancer Institute

\section{Source}

National Cancer Institute. Specify Other Performance Status Scale: Timing. NCI

Thesaurus. Code C158741.

A directive to specify a performance status scale other than one listed on the form. 